\title{
REFERENCES
}

1. G. G. Stokes, On the effect of the internal friction of fluids on the motion of pendulums, Trans. Camb. Phil. Soc. 9 (1851)

2. L. Prandtl, Ueber Reibungsschichten bei dreidimensionalen Strömung, Betz-Festschrift, p. 59 (1945)

3. W. R. Sears, The boundary layer of yawed cylinders, J. Aeronaut. Sci. 15, pp. 49-52, (1948)

4. W. Wuest, Grenzschichten an zylindrischen Körpern mit nichtstationärer Querbewegung, ZA.M.M 32, pp. 172-178 (1952)

5. L. Howarth, The boundary layer in three-dimensional flow: The flow near a stagnation point, Phil. Mag. (7) 42, pp. 1433-1440 (1951)

6. M. J. Lighthill, The response of laminar skin friction and heat transfer to fluctuations in the stream velocity, Proc. Roy. Soc. (A)224, pp. 1-23 (1954)

\section{STABILITY OF SPHERICAL BUBBLES*}

\section{BY GARRETT BIRKHOFF (Harvard Lniversity)}

The perturbation equations for a spherical bubble of radius $b(t)$ are $[1$, p. 306]

$$
b(t) b_{h}^{\prime \prime}+3 b^{\prime}(t) b_{h}^{\prime}-(h-1) b^{\prime \prime}(t) b_{h}=0 .
$$

It is the purpose of this note to give a general stability criterion for the stability of (1). Rewriting (1) in the form

$$
x^{\prime \prime}+p(t) x^{\prime}+q(t) x=0, \quad p=3 b^{\prime} / b, \quad q=-(h-1) b^{\prime \prime}{ }^{\prime} b,
$$

we consider first the formal identity

$$
\frac{d}{d t}\left\{x^{2}+q x^{\prime 2}\right\}=-\frac{x^{\prime 2}}{q^{2}}\left[2 p q+q^{\prime}\right]
$$

which is an easy consequence of (2).

Theorem 1. If $q<0$, or if $q>0$ and $2 p q+q^{\prime}<0$, then (2) is unstable. If $q>0$ and $2 p q+q^{\prime}>0$, then (2) is stable.

Proof. If $q(t)<0$, and $x(t), x^{\prime}(t)$ have the same sign for $t=t_{0}$, then they have the same sign for all $t>t_{0}$. This is evident since $x^{\prime}\left(t_{1}\right)=0$ offers the only possibility for the first sign change, and it implies $x^{\prime \prime}\left(t_{1}\right)=-q x\left(t_{1}\right)$, whence $x^{\prime}\left(t_{1}+d t\right)=-q\left(t_{1}\right) x\left(t_{1}\right) d t$ has the same sign as $x\left(t_{1}+d t\right)$. Hence $x(t)$ grows forever in magnitude; this is of course the non-oscillatory case.

If $q(t)>0$, then we are in the oscillatory case. To see this, replace (2) by the selfadjoint

$$
d\left(P x^{\prime}\right) / d t+Q x=0, \quad P=\exp \left(\int p d t\right) . \quad Q=q \exp \left(\int p d t\right) .
$$

Then we use the Bocher-Prüfer variable $\theta$, defined by $\tan \theta=-P x^{\prime} x$. Differentiating $\tan \theta$, using (4), and simplifying, we get

$$
d \theta / d t=Q(t) \cos ^{2} \theta+\frac{1}{P(t)} \sin ^{2} \theta>0 .
$$

*Received Dec. 21, 1954; revised manuscript received March 17, 1955. 
Hence (if $P(t)$ is bounded, and $Q(t)$ bounded away from zero), $x^{\prime}$ will assume the value 0 infinitely often, whenever $\Theta=\pi, 2 \pi, 3 \pi, \cdots$. Now, considering (3), we see that the amplitude of successive oscillations increases or decreases as stated in Theorem 1.

Remarks. Theorem 1 does not seem to be in the literature ${ }^{1}$, which is mostly concerned with the special case $p=0$. It does not cover cases, like that of the Mathieu equation $x^{\prime \prime}+(a+b \cos t) x=0$, in which the sign of $\left(2 p+q^{\prime}\right)$ oscillates rapidly. In the special case that $q$ is constant, then the conditions of Theorem 1 reduce to the familiar conditions $p>0$ (positive damping) and $q>0$ (positive restoring force).

Finally, in the neighborhood of a regular singular point, where $\operatorname{tp}(t)=a_{1}+\cdots$ and $t^{2} q(t)=a_{2}+\cdots$ expanded in MacLaurin series, the condition $q>0$ reduces in the limit to $a_{2}>0$, while $2 p q+q^{\prime}>0$ reduces to $2 a_{2}\left(a_{1}-1\right) / t^{3}+\cdots>0$, or (in view of $a_{2}>0$ and $\left.t<0\right)$ to $a_{1}<1$. Thus we recapture the stability conditions $a_{1}<1$ and $a_{2}>0$ proved previously $[1$, p. 308] by use of the indicial equation. In summary, the stability conditions of Theorem 1 are a direct generalization of the classical RouthHurwitz conditions.

Now, substituting into Theorem 1 from the case (1)-(2) of a spherical cavity, with negligible surface tension and cavity density, we get the stability criteria $-b^{\prime \prime} / b>0$ and $\left(-6 b^{\prime} b^{\prime \prime}-b b^{\prime \prime \prime}+b^{\prime} b^{\prime \prime}\right) / b^{2}>0$. Since $b>0$, these are equivalent to

$$
b^{\prime \prime}<0, \quad\left(b b^{\prime \prime \prime}+5 b^{\prime} b^{\prime \prime}\right)<0
$$

the second inequality is equivalent to requiring that $\left(b^{5} b^{\prime \prime}\right)$ be a decreasing function.

Evidently, the first inequality is Taylor's original criterion, that acceleration of the interface be towards the lighter fluid. The second inequality expresses the condition that there be positive damping .

Finally, we consider the general case treated recently by Plesset ${ }^{3}$. In Plesset's notation, $b=R$ and the basic equation involved can be written

$$
b_{h}^{\prime \prime}+\left(3 R^{\prime} / R\right) b_{h}^{\prime}-A b_{h}=0 .
$$

Substituting in Theorem 1, we get the two stability conditions

$$
A<0 \text { and } 6 A R^{\prime}+A^{\prime} R<0 \text {, }
$$

of which the second is equivalent to the condition that $R^{6} A$ be a decreasing function.

Historical remarks. The stability of spherical bubbles was apparently first considered by Riabouchinsky (Proc. Int. Congr. Appl. Mech., Stockholm (1930), p. 149). Riabouchinsky suggested that the spherical shape was stable when the bubble pressure was less than the ambient pressure, and unstable when it exceeded the ambient pressure. Later [Proc. Roy. Soc. A201 192, (1950)], Sir Geoffrey Taylor proposed the more accurate

1See R. Bellman, Stability theory of differential equations, New York, 1953, and references given there. However, C. T. Taam (Proc. Am. Math. Soc. 5, 705-15, Thm. 2 (1954)) has recently obtained a closely related result.

2The fact that negative damping is an important destabilizing factor in cavity collapse was pointed out by the author in July, 1952, at the Underwater Ballistics Conference in Pennsylvania State College.

3M. S. Plesset, J. Appl. Phys. 25, 96-8, formula (13) (1954). Plesset's assertion that his $G(t)<0$ implies stability, seems to be unjustified. A much more elaborate study of bubble instability has recently been given by R. H. Pennington, Tech. Res. Rept. 22 (1954), Appl. Math. and Statistics Lab., Stanford University. 
condition that there was stability when $b^{\prime \prime}<0$, and instability when $b^{\prime \prime}>0$. The need for a second condition was suggested by the author ${ }^{2}$ in 1952 ; in [1], the case of a vaporfilled bubble was treated. The present note supplies such a condition for the general case that $b$ changes by a large ratio.

\section{REFERENCES}

[1] G. Birkhoff, Note on Taylor instability, Quart. Appl. Math. 12, 306-9 (1954)

\section{ERROR BOUNDS FOR A NUMERICAL SOLUTION OF A RECURRING LINEAR SYSTEM*}

Br A. DE LA GARZA (Carbide and Carbon Chemicals Co., Oak Ridge, Tenn.)

1. Introduction. Suppose that we are given a bounded region $R, f \geq 0$, a function $\phi_{B}$ on the boundary $B$ of $R$, and point functions $f$ and $g$ in $R$, and that we are required to determine $\phi$ so that

$$
(P): \quad \partial^{2} \phi / \partial x^{2}+\partial^{2} \phi / \partial y^{2}=f \phi+g \text { in } R, \quad \phi=\phi_{B} \text { on } B .
$$

In order to calculate $\phi$ approximately, we first construct a square grid in a rectangle $S$ that has sides parallel to the coordinate axis and is large enough to contain $R$ in its interior. Then we approximate $(P)$ by a system of linear algebraic difference equations, arriving at a nonhomogeneous linear algebraic system

$$
A \phi=\eta,
$$

where $\phi$ is a vector having a component associated with each of the $N$ grid points inside $R, \eta$ is a known $N$-vector, and $A$ is a known $N \times N$ matrix. Finally, we obtain an approximate solution $\phi_{0}$ of (1), committing an error

$$
\epsilon=A^{-1}\left(\eta-A \phi_{0}\right) \text {. }
$$

A direct estimate of $\epsilon$ is given in Eq. (4) below. Our principal object is to show that $\epsilon$ can be estimated far more conveniently, though less exactly, from a properly chosen system $L$ of linear algebraic equations set up on the $M \geq N$ grid points interior to the rectangle $S$. Though motivated and illustrated by problem $(P), L$ can be constructed as soon as $A$ (with the properties listed below) is known. This method applies generally to any system (1) with those properties.

2. Estimate for $\epsilon$. We first list properties of $A=\left(a_{i j}\right)$ :

$$
\begin{gathered}
a_{i i}<0, \quad i=1(1) N ; \quad a_{i i} \geq 0, \quad i \neq j, \quad i, j=1(1) N ; \\
\sum_{j=1}^{N} a_{i j} \leq 0, \quad i=1(1) N,
\end{gathered}
$$

the inequality holding for at least one value of $i$;

*Received January 27, 1955. The work described in this paper was done under AEC Contract W-7405-eng-26. 